\title{
Towards Secure Architecture-based Adaptations
}

\author{
Narges Khakpour, Charilaos Skandylas \\ Department of Computer Science \\ Linnaeus University \\ Växjö, Sweden \\ \{narges.khakpour,charilaos.skandylas\}@1nu.se
}

\author{
Goran Saman Nariman \\ College of Science and Technology \\ University of Human Development \\ Sulaimani, Iraq \\ goran.nariman@uhd.edu.iq
}

\author{
Danny Weyns \\ Department of Computer Science \\ KU Leuven \& Linnaeus University \\ Leuven, Belgium \\ danny.weyns@kuleuven.be
}

\begin{abstract}
As any software system, a self-adaptive system is subject to security threats. However, applying self-adaptation may introduce additional threats. So far, little research has been devoted to this important problem. In this paper, we propose an approach for vulnerability analysis of architecturebased adaptations in self-adaptive systems using threat modeling and analysis techniques. To this end, we specify components' vulnerabilities and the system architecture formally and generate an attack model that describes the attacker's strategies to attack the system by exploiting different types of vulnerabilities. We use a set of security metrics to quantitatively assess the security risks of adaptations based on the produced attack model which enables the system to consider security aspects while choosing an adaptation to apply to the system. We automate and incorporate our approach into the Rainbow framework, allowing secure architectural adaptations at runtime. To evaluate the effectiveness of our approach, we apply it on a simple document storage system and on the ZNN system.
\end{abstract}

Index Terms-Self-Adaptation, Vulnerability Analysis, Security Metrics, Graph-based Threat Models.

\section{INTRODUCTION}

a) Motivations: The complexity, frequency and diversity of cyber-criminal attacks have rapidly increased over the past years. Attacks target individuals, companies, banks, the military and government agencies, causing several disruptions with severe consequences. Security breaches often happen in a system due to the presence of vulnerabilities. A security vulnerability is a weakness that can be exploited by an attacker to perform an unauthorized malicious activity. Many vulnerabilities are not discovered in systems or remain unfixed in the system after having been discovered due to a multitude of reasons, such as lack of a patch or the knowledge to fix it, cost factors, organizational preferences placing availability and usability over security. Hence, systems often contain several security vulnerabilities that are usually not isolated weaknesses forming interactions with each other, i.e. exploiting a vulnerability can facilitate the misuse of another vulnerability by the attacker. For example gaining control of a web server, would allow for serving malicious pages and in turn gaining control of more computing resources to form a botnet or conceal the attacker's identity. One of the most common techniques used to specify, discover and reason about security weaknesses and vulnerabilities is threat modeling [20], [26], [28]. Threat models are provided with analysis methods and security metrics to quantify, reason about and assess the security risks of a system while the system is operating under uncertain conditions.

Furthermore, systems are designed in a way to be able to evolve at runtime and adapt themselves to the changing requirements and the operational environment [5], [8], [16], [29], [36]. Adapting a system at runtime, however, can affect its security, e.g. by introducing new vulnerabilities and security threats. It is essential to assess the security risks of its evolution and adaptations to be able to guarantee a certain level of confidence in its security.

When a system needs to adapt because its goals may be jeopardized, several adaptations might be triggered [7], [9], [19], [27] and the system should choose and enforce one of the triggered adaptations to achieve its goals according to the business policies and preferences [12]. In addition, an adaptation can consist of several steps that are often not performed at once and the system may go through several transient configurations until it reaches the final configuration [10], [17], [18]. Consequently security has to be taken into account both during and in the resulting configuration of the adaptation process to be able to ensure a secure adaptation. To this end, each configuration of an adaptation (transient or stable) has to be studied in terms of its vulnerabilities and their interactions, prerequisites, consequences and impact to the security of the configuration and the system as a whole. Such analysis should enable the system to select those configurations and adaptations that offer the best security by minimizing the attacker's exploitation chances.

Security and vulnerability analysis of adaptations has received little attention in the past. Yuan et al. [38] provide an architecture-based approach to self-protecting systems and proposed architectural patterns to protect a system. In an extension of this work, the authors in [31] analyzed adaptations from availability point of view to be able to mitigate DoS attacks. In the network security community, researchers proposed a few approaches for security analysis of dynamic networks [1], [11], [39], [40]. However, the focus of this research is on managing the security risks of a network over time by the network administrators, rather than controlling the changes of a network. To the best of our knowledge, there has not been any research specifically on vulnerability analysis and evaluation of the security risks of a self-adaptive system that operates in an uncertain environment. 
b) This Paper: We present a general conceptual framework to perform vulnerability and security risk analysis in architecture-based self-adaptive systems. To that end, we use threat modeling techniques [20], [26], [28] to assess security risks of architecture-based adaptations. With architecturebased adaptation, we refer to adaptation that alter the structure of a running system by adding/removing/replacing components and/or connections between them. From the system architecture description and vulnerabilities specification, we generate an attack model which is used for security analysis of the system's configurations. The attack model is usually provided with (probabilistic) analysis techniques to measure a few security metrics that indicate the security risks of a selfadaptive system.

We specify an adaptation as a set of triggered adaptation rules, of which one rule is chosen to be applied in the system. An adaptation rule defines a set of action sequences performed during an adaptation process in order to achieve an adaptation goal (we explain this further in detail). Applying an adaptation rule leads the system into various transient configurations until it reaches its final stable configuration. Obviously, different adaptation rules have different security implications, as each rule usually consists of multiple configurations, each with different vulnerabilities and security risks. We propose a set of security metrics to measure the security risks of an adaptation rule based on the security assessment of its configurations. Furthermore, we measure the security level of an adaptation as a whole based on the security analysis results of its triggered adaptation rules.

We incorporate our security approach in the Rainbow [12] framework. We scan the system using vulnerability scanners to find their exiting vulnerabilities. Then, we augment the system architecture with vulnerabilities specification and automatically generate a formal attack model specified using Horn clauses. This formal model is used to generate an attack graph that describes different strategies that an attacker may take to achieve its goal. We add a new utility dimension to Rainbow, called security dimension that is obtained based on the security metrics introduced for attack graphs in the literature. This dimension is used to assess the security level of a triggered adaptation at runtime. To evaluate the effectiveness of our approach, we applied it on a simple document storage/retrieval system and the ZNN system.

c) Contributions: This paper's contributions are:

- A generic approach for vulnerability analysis and security risk assessment of architecture-based adaptations by integrating threat analysis techniques in the adaptation feedback loop. Vulnerability analysis of adaptations enables us: (i) to consider security implications of triggered adaptations at runtime while selecting an adaptation to be applied to the system, and (ii) to design more effective countermeasures to apply in the system to protect it.

- An implementation of the proposed approach in Rainbow that allows us to develop self-adaptive systems that can reason about their security dynamically and consider security preferences while designing and selecting adaptations to enforce in the system.

- An application of the security approach in two cases with experiments to evaluate the effectiveness of the approach.

This paper is structured as follows: the preliminaries are discussed in Section II. Section III introduces our running example and Section IV presents an overview of our approach. Section $\mathrm{V}$ discusses quantitative analysis of adaptations via a set of related security metrics defined based on attack graphs. Section VI describes the process of generating attack graphs from Acme (the architectural language used in Rainbow). Section VII describes the incorporation of our approach in Rainbow and an evaluation study. Section VIII discusses related work and finally Section IX concludes the paper.

\section{PRELIMINARIES}

\section{A. Threat Modeling}

Threat (or security) modeling focuses on using models to describe, analyze and discover security problems of a system. Such techniques are usually provided with quantitative analysis methods to perform security risk assessment which enables us to understand the attack consequences better and plan more effective countermeasures to defend against attacks. Quantitative analysis in threat models, for the purpose of risk assessment, is often performed based on measuring the security risk of a system using a few security metrics. Metrics can be classified into three categories: direct metrics, indirect metrics, and indicators [4]. Direct metrics are defined directly on the model and indirect metrics are defined over direct and/or indirect metrics.

Attack graphs are a class of security modeling techniques used to illustrate and analyze exploitation of security weaknesses and vulnerabilities that lead to compromising a system, i.e. they demonstrate scenarios in which an attacker can exploit known vulnerabilities of a system and compromise its security [28]. Several varieties of attack graphs have been introduced in the literature, e.g. vulnerability cause graphs [3], Bayesian attack graph [23] and compromise graphs [24]. Logical attack graphs are a type of attack graphs that considers logical causeconsequence relationships among network vulnerabilities.

Fig. 9 shows an example attack graph. The attack graphs are usually represented by two basic elements: conditions and exploits. Exploits depict vulnerabilities exploitation and conditions are either preconditions or post-conditions of exploits, i.e. they respectively show the prerequisites and the consequences of exploits. An attack graph usually has some initial states and some final states (i.e. the attacker's final goals). An attack path is a path from the initial state of the attack graph to a final goal state. There are several security metrics proposed in the literature for security risk assessment based on attack graphs, e.g. SP (Shortest Path) that considers the shortest path that an attacker can take to achieve its goal, NP (Number of Path) that counts the number of paths that an attacker can take, MPL (Mean of Paths Length) is defined as the average length of all attack paths. 


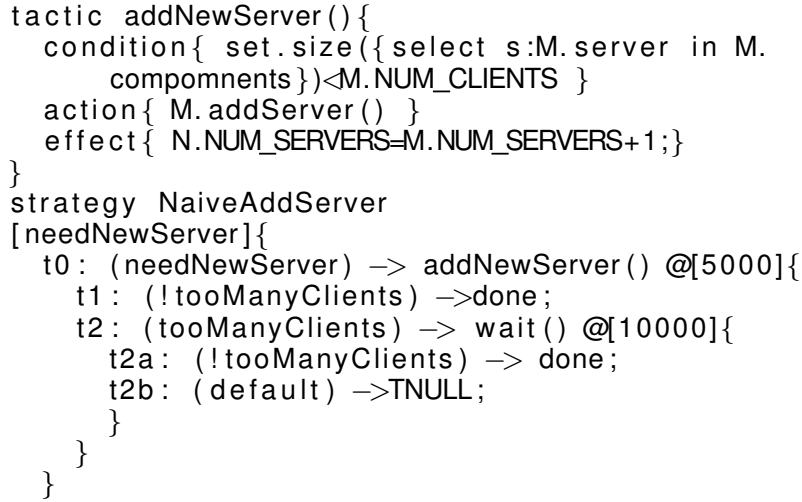

Fig. 1: A Tactic and a Strategy in Stitch

\section{B. Rainbow}

Rainbow is a framework for developing self-adaptive systems that focuses on architecture-based adaptations in order to monitor, reason about and adapt a system [12]. In Rainbow, the architectural models that represents the system architecture are kept and used at run-time to perform adaptations. Architectural models are specified in the architecture description language Acme [13], and adaptation strategies and operators are specified in Stitch [6].

In the Acme language, a system consists of a set of components and connectors. Components are primary computational elements and data stores of the system and connectors represent connections and interactions among components. A component may have ports that represent its interfaces while roles represent the interfaces of connectors. Components can additionally contain properties which are represented by a name, an optional type and a value. Fig. 6 shows an Acme specification.

Stitch contains three basic elements: operators, tactics, and strategies. Operators refer to single atomic steps taken during the adaptation process. A tactic comprises of a condition, an action and an effect block. The condition block corresponds to the precondition under which the tactic will be triggered, the action block is a set of actions composed by operators that will be applied to the system and the effect block is the affect of executing the tactic on the system's model. A strategy is a sequence of conditional tactic executions. It is characterized by a tree of condition-action-delay decision nodes. The applicability of the tactic is specified by a predicate evaluated over the architectural model for strategy selection. Fig. 1 shows a tactic and a strategy.

When an adaptation is triggered, the quality of triggered strategies is evaluated in terms of the utility preferences that specify business priorities over the quality dimensions of a system. A quality dimension is a business concern related to the system. Every such dimension defines a utility function that is mapped to an architectural property of the system (i.e. a property about the architecture). For each tactic, an impact vector identifies the cost and the benefit of executing this tactic over each of the quality dimensions.

\section{RUNNING EXAMPLE}

As a running example, we consider the document storage/retrieval system shown in Fig. 2. The frontend component is responsible for handling the users input, and the account management component (accountManager) takes cares of the registration/logging in to the system as well as keeping a list of the active users. The document management (documentManager) handles searching for, storing and uploading a document. The database manager (databaseManager) handles all interactions to the database which stores both the user information and the location and access rights for documents. The file manager component (fileManager) that handles the storage and retrieval of the actual document files. Finally a guardian component can be enabled to improve the response time of the system by blocking all writes to the database and limiting each user's session reads. The guardian does not appear in the figure as it is disabled by default.

The above system might need to adapt its behavior to better cope with non-functional requirements. For instance, if the database load becomes too heavy to cope with the users needs in terms of response time, it might be required to separate the accounts and documents in separate databases and then employ two database manager components, one for each, to increase throughput. Likewise, if a lot of document accesses are being requested, two file manager components could be employed.

When this adaptation occurs, adding or removing components or interaction between such components affects the system's security both during the adaptation process and afterwards. Assume that a load balancer component is added that interacts both with (fileManager) and (databaseManager). If an exploit allows for user credentials to be leaked from the database manager, the load balancer serves as an additional entrypoint to enable attacks to the file manager. We therefore need to ensure that during and after adaptation the system's security level is kept as high as possible by selecting a secure adaptation strategy as well as a secure final configuration.

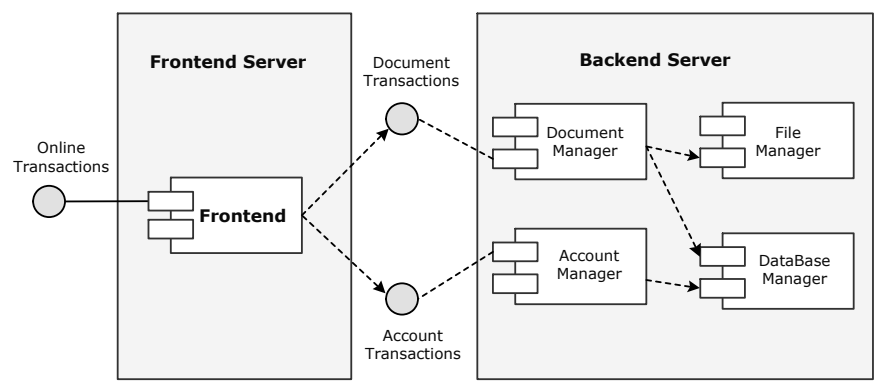

Fig. 2: A Document Storage/Retrieval System

\section{Security-Aware Feedback Loop}

We enhance the MAPE-K (Monitor-Analyze-Plan-ExecuteKnowledge) feedback loop with security analysis as shown in Fig. 4. In the monitoring phase, system vulnerabilities are regularly gathered by scanning the system using vulnerability scanners and the results are stored in the knowledge base. We 
augment the architectural model of the system with security information, which is maintained at runtime to generate an attack model that allows assessing the security risks of a system configuration. An attack model represents the attacker's strategies to achieve a specific goal in a specific architecture.

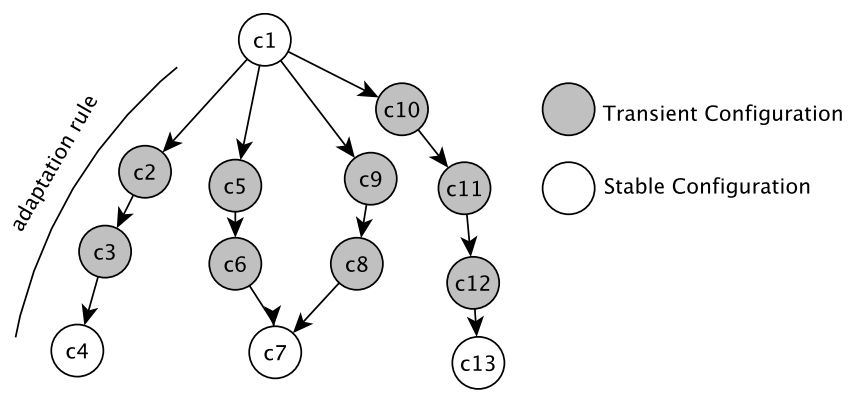

Fig. 3: A Typical Adaptation Process

When an adaptation is required, a set of adaptation rules are triggered, among which one is chosen to be applied to the system. As explained in the introduction, an adaptation rule is a sequence of actions performed by the system, see Fig. 3. Applying an adaptation rule leads the system to go through several transient configurations until it reaches the final configuration. In the analysis phase, the security risks of the triggered adaptation rules are assessed and the most suitable adaptation, in terms of security risk and/or other preferences, is selected to be applied to the system.

To assess the security risk of an adaptation rule, we first evaluate the security risks of all of its configurations individually, i.e. we consider the security status of all the transient and stable configurations in our evaluation. To this end, an attack model is generated for each configuration separately and we use a set of security metrics defined for that model to assess the security risks of the configuration. Afterwards, we use newly introduced indirect security metrics to assess the security of an adaptation rule. Similarly, the security of an adaptation (i.e. a set of triggered adaptation rules) is assessed using novel security metrics defined for adaptation evaluation.

\section{Quantitative Security Assessment of ADAPTATIONS}

In this section, we assume that the attack model of an adaptation is specified as a set of attack graphs where each graph shows the threat model of an individual configuration of the adaptation (i.e. it can be a transient configuration or a stable configuration). Then, we introduce a few security metrics for risk assessment of adaptations that are defined based on attack graphs. To analyze security of an adaptation rule, we first assess the security of each of its individual configurations separately using a few security metrics. Then, we define new security metrics to measure security of an adaptation rule based on the security metrics of its individual configurations. Finally, the security level of an adaptation is determined based on the security metrics of its adaptation rules. a) Security Assessment of Individual Configurations: As mentioned above, for each individual configuration, we produce an attack graph from its architecture and vulnerabilities descriptions and use this attack graph to assess its security risks. The security metrics defined based on attack graphs can be classified into various path metrics, non-path metrics, probabilistic metrics, Bayesian network-based metrics and others in [28]. We selected three path-based and probabilistic metrics shown in Table I to assess the security level of a configuration. A discussion on the justification of the above metrics' definitions can be found in [28]. The definitions of the first two metrics are quite obvious.

AGP (Attack Graph Probabilistic) [35] is a probabilistic metric which is defined for nodes in an the attack graph and represents the probability of reaching a specific node in the attack graph via several multi-step attacks. This metric is defined based on the severeness of individual vulnerabilities, e.g. according to the Common Vulnerability Scoring System (CVSS) ${ }^{1}$ score in addition to the causal relationships among vulnerabilities encoded in the attack graph. CVSS is a framework for categorizing and rating the severity of security vulnerabilities in software. The AGP of an attack graph is defined as the average of the AGPs of all the attacker's goals.

TABLE I: Configuration-Level Security Metrics

\begin{tabular}{|l|l|}
\hline Name & Description \\
\hline MPL [15] [22] & Average length of all attack paths to the attacker's goal \\
\hline NP & Number of attack paths \\
\hline AGP [35] & Average AGP of all the attacker's goals \\
\hline
\end{tabular}

b) Security Assessment of Adaptation Rules: For each configuration-level security metric presented in Table I, we define a corresponding adaptation rule-level indirect metric. Each adaptation rule-level metric shown in Table II starts with $\mathrm{P}$ followed by its corresponding configuration-level metric. In this table, $n$ indicates the number of configurations of the adaptation rule and Avg is the ordinary average function.

TABLE II: Adaptation Rule-Level and Adaptation-Level Security Metrics

\begin{tabular}{|l|l|l|l|}
\hline \multicolumn{2}{|c|}{ Adaptation Rule-Level Metrics } & \multicolumn{2}{l|}{ Adaptation-Level Metrics } \\
\hline \hline Name & Formula & Name & Formula \\
\hline PMPL & $\operatorname{Avg}_{i=1}^{n} M P L_{i}$ & RMPL & $\operatorname{Avg}_{i=1}^{m} \phi_{i} * P M P L_{i}$ \\
\hline PNP & $\operatorname{Avg}_{i=1}^{n} N P_{i}$ & RNP & $\operatorname{Avg}_{i=1}^{m} \phi_{i} * P N P_{i}$ \\
\hline PAGP & See Equation (1) & RAGP & {$\left[\sum_{i=1}^{m} \phi_{i} * P A G P_{i}\right] / m$} \\
\hline
\end{tabular}

PAGP defines the probability of a successful attack by the attacker (i.e. reaching the attacker's goal in the attack graph) in at least one of the configurations of the adaptation rule. The probability that a particular configuration in a path is successfully attacked depends upon its previous configurations, as we assume that when the attacker takes control over the system,

\footnotetext{
${ }^{1}$ https://www.first.org/cvss/
} 


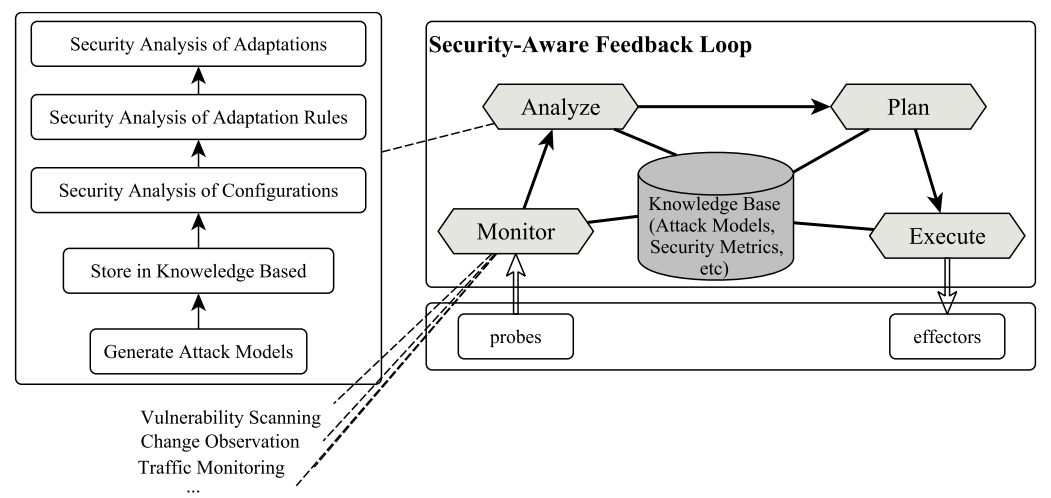

Fig. 4: Security-Aware Feedback Loop.

the system's behavior won't be the ordinary expected behavior and we can expect any kind of undesired behavior. Hence, the attack models will not represent the attacker's strategies anymore. So, if an attack occurs in any of the intermediate configurations, it is evident that the attack has been successful for that adaptation rule. As a result, whatever happens in the succeeding configurations can be ignored.

Building upon the above discussion, if a particular configuration is successfully attacked, it is clearly implied that previous configurations have not been successfully attacked. Clearly, the probability of attack success and attack failure of each configuration in a path are the complement of each other. Let an adaptation rule $\pi$ consist of the sequence of configurations $c_{1}, c_{2}, \ldots, c_{n}$ and $\mathrm{AGP}_{c_{i}}$ denote the configuration $c_{i}$ 's attack success probability. A configuration $c_{i}$ can be attacked, if it had not been attacked in any of its previous configurations $c_{j}, 1 \leq j<i$. Hence, the probability of $c_{i}$ being attacked is defined as $\prod_{j=1}^{i-1} \overline{\mathrm{AGP}_{\mathrm{c}_{\mathrm{j}}}} * A G P_{c_{i}}$ where $\overline{A G P_{c_{j}}}$ shows the probability of attack failure in $c_{j}$. We define the $P A G P$ of $\pi$ as the following formula:

$$
P A G P_{\pi}=A G P_{c_{1}}+\sum_{i=2}^{n}\left[\prod_{j=1}^{i-1} \overline{A G P_{c_{j}}} * A G P_{c_{i}}\right]
$$

Formula (1) states that the probability of a successful attack on $\pi$ is the summation of the attack success probabilities of its configurations (See [30] for more details).

c) Security Assessment of Adaptations: We design indirect metrics to evaluate security risk of an adaptation based on the security metrics of its adaptation rules. Table II shows the list of adaptation-level indirect metrics, where $m$ is the number of its triggered adaptation rules, $\phi_{i}$ is the probability of applying the $i$-th adaptation rule and $\sum_{1<i<m} \phi_{i}=1$. We multiply the probability of an adaptation rule being applied with its security metric result to obtain its security risk. If an adaptation is more probable to be triggered, its security risk has a higher impact on the adaptation's security level. Adaptation-level metrics start with $\mathrm{R}$ followed by their corresponding configuration-level security metric.

\section{Security Enhancement of Rainbow's ADAPTATIONS}

This section discusses the instantiation and incorporation of our approach in Rainbow.

\section{A. Security Dimension in Rainbow}

When an adaptation is triggered in Rainbow, each strategy available to the system is evaluated, and the best strategy is selected. The best strategy is determined based on the impact of that strategy on the system's quality dimensions calculated by aggregating the impact of each of the strategy's tactics and scoring them over the quality dimensions. Once the best strategy is selected, it gets executed. We introduce a new utility dimension called security dimension that is defined based on the configuration-level security metrics.

To calculate the security dimension of a configuration which is mapped to an architectural property, we need to generate the attack graph of the architecture corresponding to that configuration first. To this end, we exploit the Acme language to specify the system architecture and its security flaws (Section VI-B) which enables us to generate a formal specification of the system and its vulnerabilities semi-automatically in terms of Horn clauses (Section VI-C). From the formal specification, we generate an attack graph that represents the attacker's strategies in the configuration (Section VI-D). The generated attack graphs then serve as input for security analysis of the adaptation process. We use the set of security metrics defined in Table I to asses security risk of an architecture.

In Rainbow, each strategy is composed of a number of different tactics which aim to achieve the strategy's goal. The tactics are arranged in a tactics tree whose distinct paths correspond to the adaptation rules, i.e. an adaptation rule is then formed as a list of those configurations from the starting configuration (the root of the adaptation tree), to the leaves of the tree which comprise of either done or TNULL, with done signifying success and TNULL signifying failure. Each of the nodes in the adaptation tree is associated with a new configuration (architecture) which is computed by applying the effects of the tactic corresponding to the 
visited node to the architecture of its parent node. We rely on the Rainbow's approach for calculating the security metrics of an adaptation rule that we believe is an instantiation of our metrics definitions introduced in Table II. The process of evaluating security of a strategy's tactics is depicted in Fig. 5.

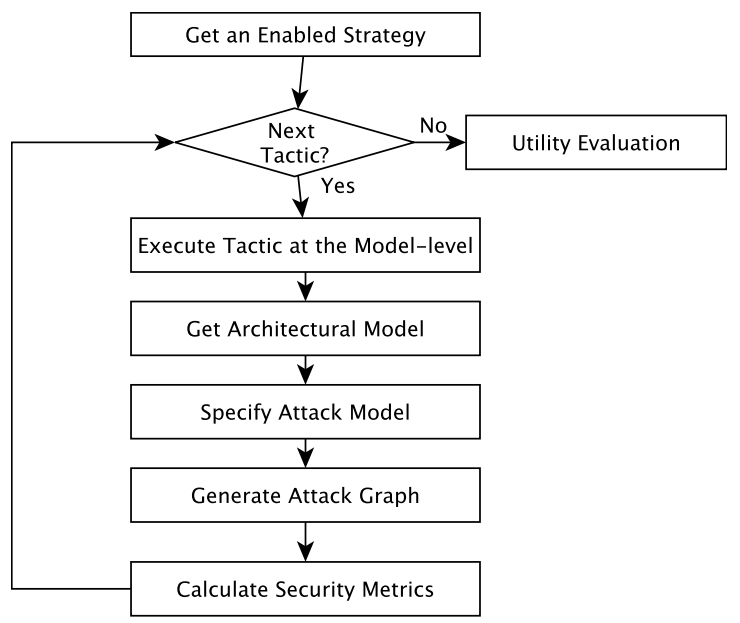

Fig. 5: Security Evaluation of a Strategy's Tactics

\section{B. Vulnerability Specification of Components in Acme}

In order to specify system's vulnerabilities, we make use of the Property notion provided by Acme. We consider two types of components: internal components that we can access their internal code and external components that are in principle black-box components. In order to specify multiple vulnerabilities in each component, we make use of the Sequence type of properties in Acme. Therefore vulnerabilities are specified as a sequences of Strings. Internal component vulnerabilities are defined using the vulnerable method or function and an id that specifies the vulnerability type in pairs. Similarly exVulns defines external vulnerabilities, corresponding only to the vulnerability type. The vulnerability type is obtained from existing databases such as CWE (Common Weakness Enumeration $)^{2}$ that is a category system for security-related flaws in software and assign an identifier to each weaknesses, e.g. CWE_119 shows a buffer overflow problem.

Example 1: Fig. 6 shows the specification of the databaseManager (an internal component) and database (an external component) in our running example. The databaseManager component has a SQL injection vulnerability in its executeQuery method associated with CWE_89. There also exists a vulnerability allowing a buffer overflow related to CWE_119 in the database. These internal and external vulnerabilities can be associated with a CWE reference, however, internal vulnerabilities provide more information which enables us to be more precise in our analysis of internal components.

\section{Attack Model Specification}

Given the augmented Acme model, we generate a formal attack model semi-automatically. The attack model comprises

\footnotetext{
${ }^{2}$ https://cwe.mitre.org/
}

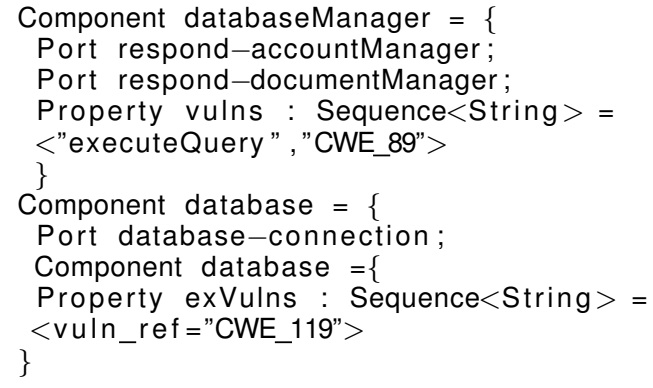

Fig. 6: An Acme Example

of (i) the system architectural description (i.e. specification of components and their interactions), (ii) specification of vulnerabilities, and (iii) the exploitation and propagation semantics of vulnerabilities. We will formalize the attack model using Horn clauses and discuss it in this section.

a) Horn Clauses: We represent a predicate by $p\left(t_{0}, \ldots, t_{n}\right)$ where a term $t_{i}, 0 \leq i \leq n$ is either a constant or a variable. A Horn clause is a rule-like formula defined on predicates and is of the form

$$
p_{0}\left(t_{0,0}, \ldots, t_{0, m_{0}}\right), \ldots, p_{k}\left(t_{1,0}, \ldots, t_{1, m_{k}}\right) \vdash q\left(t_{0}^{\prime}, \ldots, t_{n}^{\prime}\right)
$$

that informally states if for all $0 \leq j \leq k$, the predicate $p_{j}$ holds, then $q$ will hold. The left hand side of this rule is called body and $q$ is called the head.

When a Horn Clause is expressed in a non-propositional form, all its variables are universally quantified. For example, $\quad p(x, y), q(x) \vdash r(x, y)$ is equivalent to $\forall x, y \cdot p(x, y) \wedge q(x) \Longrightarrow r(x, y)$. In the logic programming, $p(x, y), q(x) \vdash r(x, y)$ is written as $\mathrm{r}(\mathrm{x}, \mathrm{y}):-\mathrm{p}(\mathrm{x}, \mathrm{y}), \mathrm{q}(\mathrm{x})$ which is the notation that we will follow in this paper.

b) Architecture Description: Table III shows the transformation from an ACME Model to predicates partially. To generate the architecture's formalization from Acme specification, we describe each internal component $\mathrm{c}$ in the Acme specification by the predicate isComponent(c). The predicate methodlnvocation (method,from, to) states that the component from can invoke the method method of component to. The elements of ports, roles and attachments are used to generate the methodlnvocation predicates: from is the first role of a connector between from and to combined with the attachment, to is the second role and method is extracted from the connector's name. Attachments provide a mapping from Ports to Roles in the architectural model.

We use the predicates canlnvoke(from,to,method) to model interactions among components. The predicate canInvoke(from,to,method) holds either (i) if there is a direct method invocation between two components (i.e. methodlnvocation(from,to,method) holds), or (ii) there is an invocation between them through another component in a transitive manner, i.e. component from can invoke a method of an intermediate component comp and comp can invoke method of to.

An external component ext in the Acme specification is described by the extComponent(ext) predicate. External com- 
ponents can provide external interfaces (that allow them to be called externally i.e. by connecting to an outward facing port) specified by the exInterface(ext) predicate or are called from internal components described by the exInvocation(ext,comp,m) predicate. Interactions between internal and external components are specified by the exInvocation(ext,comp,m) predicate where the internal component comp invokes the method $\mathrm{m}$ which interfaces with the external component ext.

c) Specification of Vulnerabilities: An existing vulnerability in an internal component is defined using a predicate compVulnExists $(\mathrm{c}, \mathrm{m}, \mathrm{v})$ where $\mathrm{c}$ is an internal component and $\mathrm{m}$ is a method containing the vulnerability $v$. For external vulnerabilities, the predicate exVulnerability $(e x t, v)$ specifies that the external vulnerability $\mathrm{v}$ is present in ext.

Example 2: The following specification is generated for the Acme specification in Example 1:

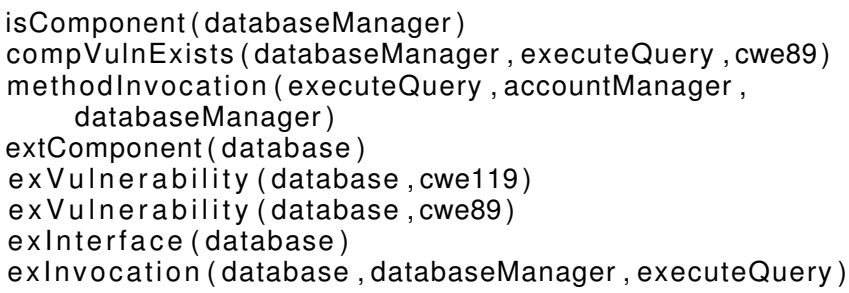

d) Exploitation and Propagation of Vulnerabilities: For a vulnerability to be exploited, a set of prerequisites should hold and exploiting a vulnerability will lead to a set of consequences. We specify the list of vulnerabilities manually as described above. An internal component is compromised if the attacker controls that particular component denoted by the predicate attackerLocated(comp), or there exists a transitive relationship so that an already compromised component comp' can invoke a vulnerable method of that component. For instance the frontend component in the running example can exploit an authentication bypass vulnerability in the login method to compromise the account manager, which then enables compromising the database manager via the executeQuery method. An external component can be compromised if either (i) it exports an external interface that contains a vulnerability, or (ii) it can be invoked from a compromised internal component in a method that can cause an external vulnerability, and that vulnerability is present in the external component. The above rules are specified in Fig. 7

A vulnerability exploitation is then specified by specific rules for basic and more complex attacks. Fig. 8 depicts the rules describing a command injection attack, enabled by internal or external components, as well as two more complex attacks building on command injection, privilege escalation and document deletion which then leads to a compromise. To be able to delete a document, the attacker needs to combine two simpler attacks to facilitate a more complex goal. Attacks consequences are tied to one or more aspects of three security aspects of confidentiality, integrity or availability. For instance, the integrityCompromised rule specifies that the integrity of a component is compromised when an attacker can delete a document using that component. This type of specification of

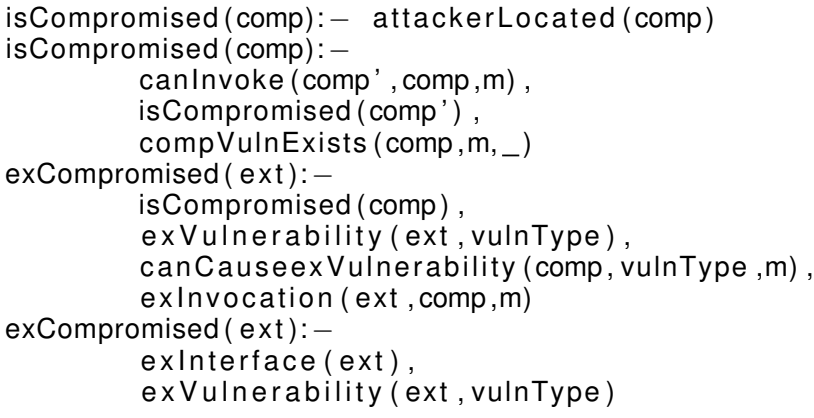

Fig. 7: Interaction and Compromise Propagation Rules

consequences based on confidentiality, integrity and availability violation permit a flexible way to specify when a system is to be considered no longer safe to operate. We utilize the systemDown rule for this purpose which can be crafted based on the aspects compromised but specified as much or as little as needed by the design, i.e. a very strict specification would assume (as we do) that a system is to be considered down if any quality in any component has been compromised. A more practical design would be to target specific aspects, i.e. integrity in the key components of the system, e.g. the database.

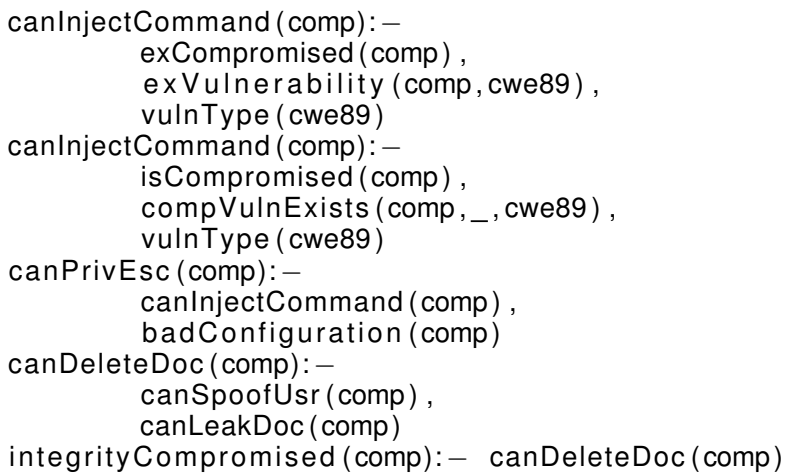

Fig. 8: Vulnerability Exploitation Rules

\section{Attack Graph Generation}

An attacker's goal can vary from compromising a specific component to shutting the whole system down. A goal is formalized as a predicate whose satisfaction indicates that it has been achieved by the attacker. For instance, the predicate systemDown(System) in our example shows that a system is unavailable due to several reasons such as a DoS attack. Given an attack model and the attack goal, we use MulVAL (Multihost, multistage Vulnerability Analysis) [26] to generate an attack graph. MulVAL is a framework for modeling and reasoning about the interaction of software bugs with system and network configurations. It uses Datalog as its modeling language and supports AGP for probabilistic security risk estimation of the produced attack graph. 
TABLE III: ACME Elements to Predicates Mapping

\begin{tabular}{|c|c|}
\hline ACME Elements & Predicates \\
\hline Component c & isComponent(c) \\
\hline Component ext $=\{$ Property isExternal $=$ true $;\}$ & extComponent(ext) \\
\hline Connector $m=\{$ Role from; Role to; $\}$ & methodlnvocation (m,from,to) \\
\hline Connector $m=\{$ Role from; Role ext $;\}$ and Component ext $=\{$ Property isExternal $=$ true $;\}$ & exInvocation(ext, from,m) \\
\hline Component ext $=\{$ Property isExternal $=$ true $;$ externallnterface $=$ true $;\}$ & exInterface (ext) \\
\hline Component $\mathrm{c}=\{$ Property vulns: Sequence $<$ String $>=<\mathrm{m}, \mathrm{v}>\}$ & compVulnExists $(c, m, v)$, vulnType(v) \\
\hline Component ext $=\{$ Property exVulns: Sequence $<$ String $>=\{v\} ;\}$ & exVulnerability (ext,v), vulnType(v) \\
\hline
\end{tabular}

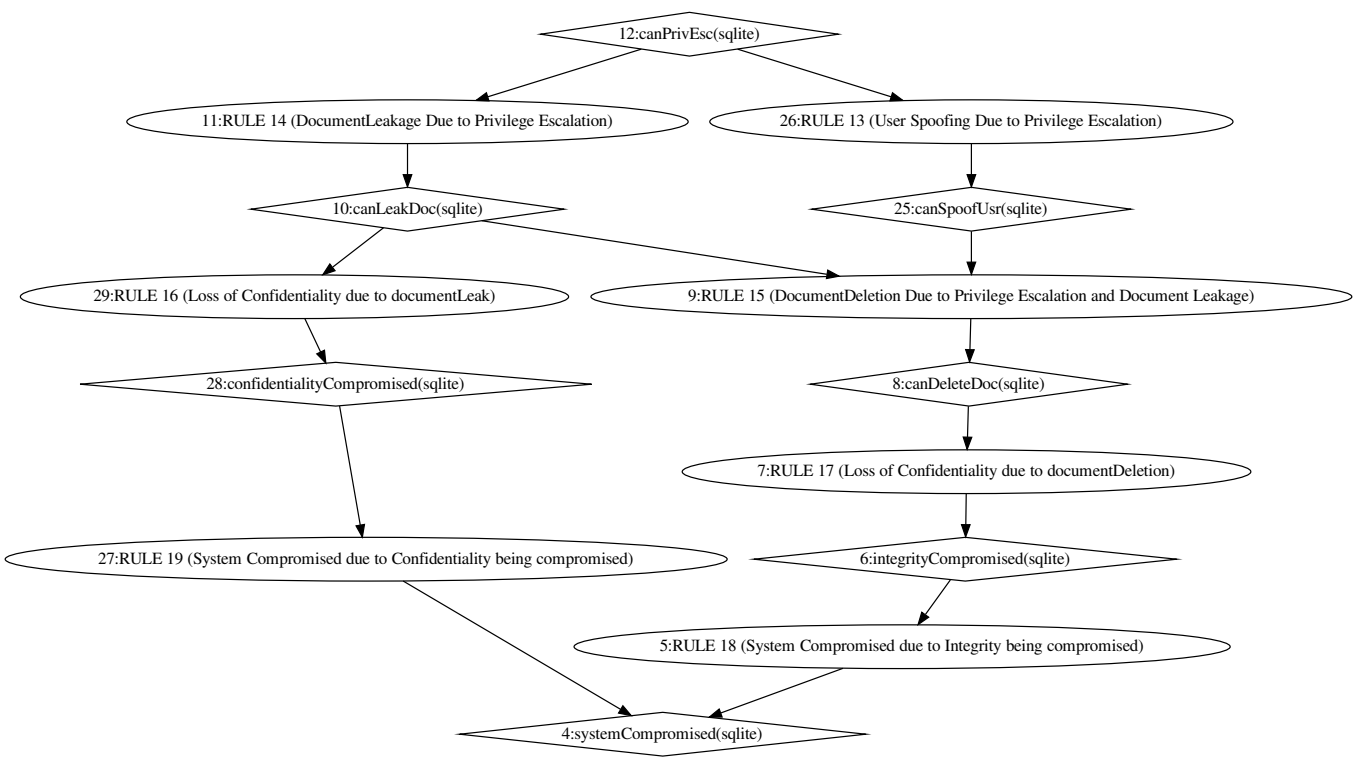

Fig. 9: The Generated Attack Graph

Example 3: Fig. 9 depicts a part of the attack graph generated for the running example, with systemDown as its attack goal. In this part of the graph, we can see that the system is considered non-functional when either the integrity or confidentiality of the database component is compromised. Confidentiality can be compromised by a privilege escalation followed by a document leakage, while integrity is a bit harder to compromise and requires a combination of document leakage and user spoofing.

\section{IMPLEMENTATION AND EVALUATION}

This section discusses the implementation of our approach in Rainbow as well as an evaluation study.

\section{A. Implementation}

Fig. 10 shows a high-level view of the implementation of security analysis. The implementation consists of four basic

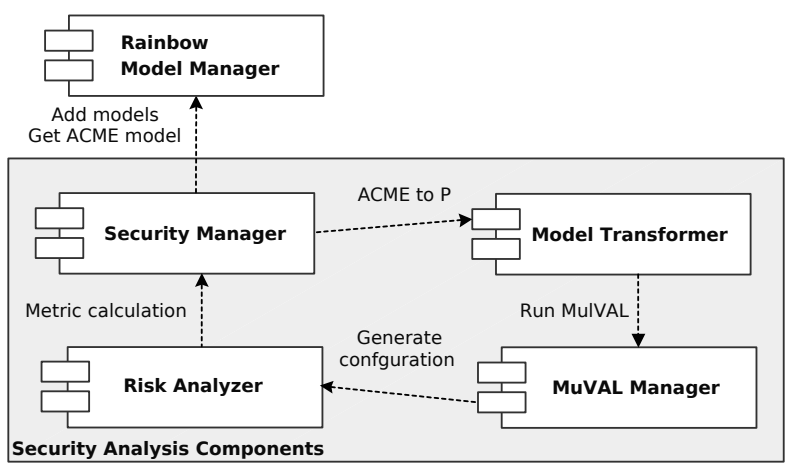

Fig. 10: The Component Diagram of Security Risk Assessment

components: model transformer, MulVAL manager, risk analyzer and security manager. The model transformer is responsible for transforming a run-time or textual Acme model to the 
corresponding Datalog input. The MulVAL manager ${ }^{3}$ supports logical attack graph generation and probabilistic security assessment of nodes in the attack graph. Each configuration is stored into the risk analyzer. The risk analyzer provides a set of implemented security metrics that can be used for security risk analysis. This component builds a configuration graph (see Fig. 3) and calculates the configuration-level metrics on that graph. Then these metrics can be used as utility functions in rainbow to evaluate the security dimension. The security manager component drives the security analysis process, acting as an entrypoint, and controller for the process and an interface with Rainbow's model and adaptation manager components.

\section{B. Evaluation}

a) Cases: To evaluate our approach, we conducted a set of experiments on two cases: the document storage/retrieval system and $\mathrm{ZNN}^{4}$. $\mathrm{ZNN}$ is a well-known self-adaptive exemplar that provides a news service that serves news content to clients. From an architectural perspective, ZNN comprises of a set of servers controlled by a load balancer that manages the client connections. The aim of self-adaptation is to balance the load among the servers and provide fast response times, regardless of dynamic user requests. Architecturally, adaptation operations include enabling a server, disabling a server, and increasing or decreasing fidelity. For our document storage/retrieval system, we only allow adding/removing frontends and enabling a guardian component to reduce response time by limiting reads and blocking writes to the database.

We used Progpilot ${ }^{5}$ to scan ZNN's source code vulnerabilities which is a static analysis tool for security. We identified 9 classes of internal vulnerabilities related to functions in ZNN source code. We also used OpenVAS ${ }^{6}$ to detect its external vulnerabilities that discovered 45 classes of external vulnerabilities. The generated attack graphs for ZNN's configurations contain approximately 300 to 400 nodes each.

b) Effectiveness: To determine the effectiveness of the approach, we measure the security level of a system in each stable configuration after executing the system for a number of adaptation steps, with and without security analysis being enabled. In Fig. 11 and Fig. 12, we refer to settings as follows: SA corresponds to security analysis and the value that corresponds to the weight placed to the security dimension, e.g. ZNN-SA-0.8 reads as ZNN with security analysis and a security dimension weight of 0.8 . The figures show the measurements of the security metrics after executing the system for a number of adaptations, i.e. in the stable configurations. In these figures, the higher the value of NP, the less secure the system will be, as the attacker will have more available paths to attack the system. Furthermore, a low AGP value entails a more secure system, as it indicates that the attacker has a smaller chance to attack the system successfully. Similarly, lower values of MPL indicate that the attacker is required to

\footnotetext{
${ }^{3}$ MulVAL manager is a thin wrapper on top of the MulVAL implementation. ${ }^{4}$ https://github.com/cmu-able/znn

${ }^{5}$ https://github.com/designsecurity/progpilot

${ }^{6} \mathrm{http}: / / \mathrm{www}$. openvas.org/
}

perform fewer steps to succeed in his goal and hence is less secure.

As a threat to validity, we acknowledge that we only performed a relative small number of experiments and further study with more complex systems is required to derive stronger conclusions. Nevertheless, from the results of our experiments we can observe that setting a medium to high weight to the security dimension increases the security level of the system as demonstrated in Fig. 11 or Fig. 12. In addition, we observe that even a fairly low weight of 0.2 will result in adaptations that are at least as secure as the case where no security analysis is applied (See Fig. 11 or Fig. 12).

By analyzing the selected adaptations in the document storage/retrieval system, we notice that when security is of high concern, the adaptation selected is the one that performs no architectural changes to the system, thus the first adaptation will always opt to execute enabling the guardian. Enabling the guardian, incurs a welcome side-effect of limiting an attacker's writing capabilities to the database. The only other option would be to add a front-end to the system which would be detrimental to its security since it enables a multitude of new avenues of attack, as can be seen from the number of paths measurement in Fig. 11 (NP diagram). Given that the system only has two options to adapt to: adding a frontend which would incur a significant security penalty or enabling the guardian which performs no architectural adaptation. We can easily see that the system selects the most secure of the two, i.e. adding a frontend allows for further pathways for an attacker to exploit, whilst enabling the guardian performs no architectural change and in fact limits the attacker's interaction with the database

In $\mathrm{ZNN}$, there are only four servers available in our version of the system. As a result, after enabling all four servers, no further servers can be enabled by applying an adaptation. Thereby, the system will converge to the same final architecture in order to satisfy the quality dimension of response time. Nevertheless, when a significant weight is placed upon the system's security, the selected adaptation is to alternate between executing two opposing strategies of decreasing and increasing fidelity, rather than enabling a server which would reduce the average response time but would greatly impact security as seen by the corresponding figure for ZNN in Fig. 12. When the value of other quality dimensions is sufficiently low, the system will opt for adaptations that will not compromise security, even when low priority is placed in security. One such adaptation is adaptation 2-3 in ZNN-SA0.2 shown in Fig. 12 which corresponds to opting to lower fidelity rather than enabling a new server.

c) Computational Complexity: The complexity of attack graph generation for a single configuration is $O\left(m^{2} \log m\right)$ where $m$ is the number of components [25], [34]. With $n$ the total number of tactics of the triggered strategies, the complexity of the whole attack graph generation process for an adaptation is $O\left(\mathrm{~nm}^{2} \log \mathrm{m}\right)$. The complexity of security metrics measurement is basically the complexity of graphtraversal algorithms which is polynomial in terms of the size 


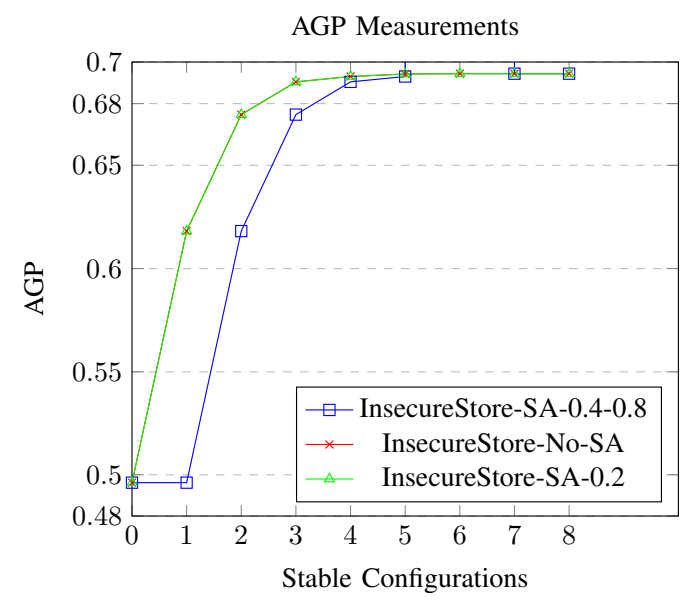

MPL Measurements

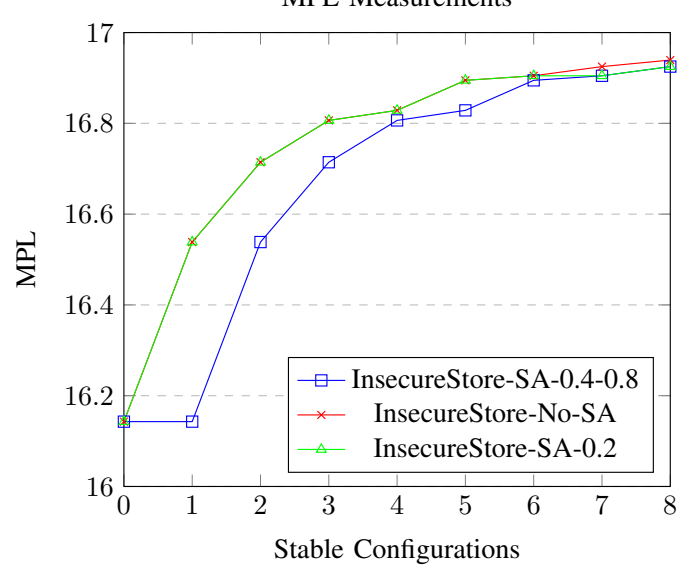

Number of Paths Measurements

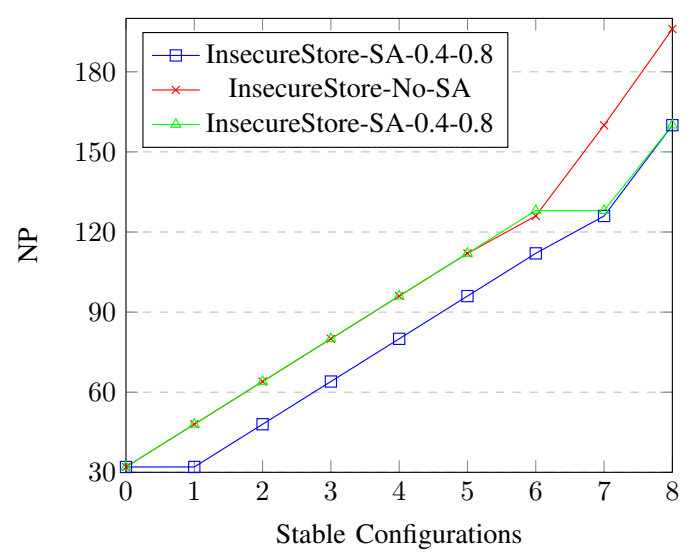

Fig. 11: Metric Measurements for the InsecureStore

of the attack graph. Since the final configurations are usually exposed to the attackers for a longer period, their security is of a greater importance compared to the transient configurations. To enhance the performance, one can only assess the security risks of final configurations instead of evaluating the security risks of all the transient configurations. This will practically reduce the complexity of analysis to $O\left(\mathrm{~km}^{2} \log \mathrm{m}\right)$ where $k$ is usually a very small number and represents the number of possible final configurations.
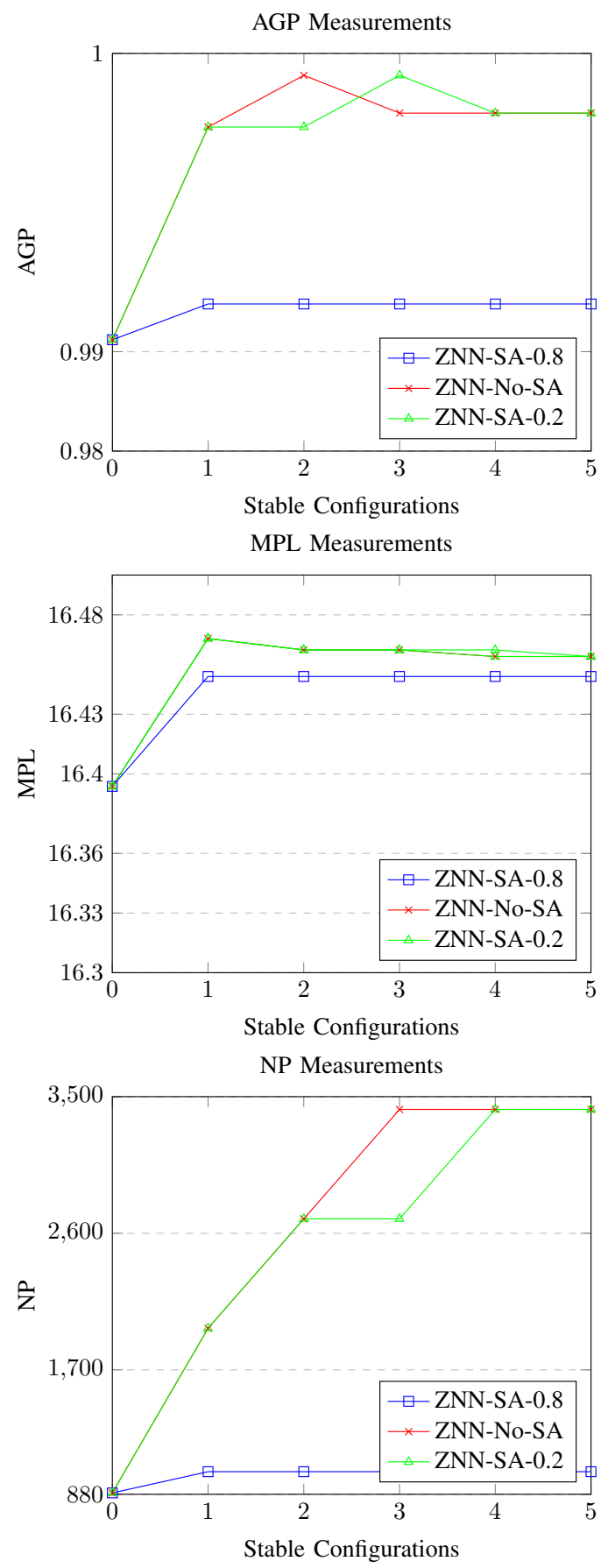

Fig. 12: Metric Measurements for ZNN

\section{RELATED WORK}

Self-protection refers to the ability of a system to dynamically adapt its behavior at runtime to protect itself against threats and attacks. The authors in [37] surveyed the existing work and proposed a taxonomy for self-protecting systems. Yuan et al. [38] provide an architecture-based approach to self-protection. They provide several architecture adaptation patterns that can be used to detect and mitigate security weaknesses in web applications. The patterns are implemented 
in a well-known exemplar SAS, by employing the Rainbow framework. In an extension of this work [31], DoS-related tactics are composed to mitigate DoS attacks and the utilitybased reasoning provided in Rainbow is used to choose the best strategies to apply in the system. In contrast to the above approaches, we take into account the security risks of components' vulnerabilities while selecting a strategy to apply in the system. We introduce a new utility dimension to assess security risks of different tactics on the system, which enables the system to consider the security implications of adaptations on the whole system's security and applies the adaptations that are more secure according to the security policies. In [2], the authors employ a MAPE-K reference model to dynamically negotiate and deploy security policies in mobile devices and use dynamic software product lines to adapt the security level of the application at runtime to provide adaptive security. This work is not concerned about security analysis of adaptations while we focus on security risk assessment of adaptations.

Lamprecht in his thesis [21] provides a solution and methodology to support runtime security adaptation in non-adaptive legacy system while addressing the trade-off between performance and security. Based on an offline analysis, he dynamically creates policies to trade-off performance and security. He mainly focuses on applying and adapting cryptographic algorithms to ensure security in terms of privacy and the main concern is performance. Our approach targets adaptive systems with the aim of selecting the most secure possible adaptation.

In [33] Tun et al. propose an approach to specify adaptive security under partial knowledge of the environment that can be later enhanced as more knowledge becomes available at runtime forming an adaptive specification of security. Requirements are split into episodic and run requirements. Episodic requirements can then be adjusted at runtime if they are violated to satisfy run requirements. Adaptation is done at runtime via input approximation which is facilitated by machine learning techniques. Our approach assumes perfect knowledge of the system's architecture and vulnerabilities and security policies used to do risk assessment are fixed. We are not concerned with adapting security policies themselves and rather support the system to assess adaptations from security point of view and apply the more secure adaptations.

Security risk analysis of dynamic systems is another related area of research that has been receiving some attentions recently. HARM (Hierarchical Attack Representation Model) [14] is a multi-level modeling introduced to improve scalability of modeling languages to assess the security risks of a large-scale network. In HARM, a model consists of several layers and the layers can be modeled using different security models. In contrast to our approach, HARM modeling is more modular and scalable. To handle dynamic systems, THARM (Temporal-HARM) [39], [40] has been introduced as an extension of HARM, to capture the changes of a network and their impact on the system security over time. The security model of a dynamic network is defined as a sequence of HARM snapshots and a few security metrics are defined for the HARM snapshot sequences, in a similar fashion to ours.
In contrast to our approach, T-HARM is neither concerned with security risk assessment and management of changes, nor considers the security risk of adaptation steps.

Dynamic Bayesian Network has been used to assess security of networks by considering the evolution of vulnerabilities in [11]. The authors used attack graphs and CVSS to construct dynamic bayesian networks. This work does not consider the structural changes and focuses only on the dynamic security aspects of a network.

Almohri et al. [1] introduced a probabilistic model to measure security risks in dynamic networks, in particular, to check the likelihood of successful attacks in the presence of uncertainty about the configurations of the network. They used this model to optimally deploy security controls in a dynamic network. While we try to manage adaptations by checking their security impact on the system, they are concerned with operating securely in an uncertain environment.

Online security risk assessment focuses on monitoring security risks of a system at runtime. Ten et al. proposed a framework for online security risk management of power systems in [32] that uses a database of attack patterns described using attack trees. This framework contains different components for real-time monitoring, anomaly detection, impact analysis and mitigation strategies to provide self-protection. Since we generate the attack graphs automatically, our approach is more automated compared to [32]. In addition, we consider the security risks of adaptation phase while [32] is not concerned with this problem.

\section{CONCLUSION}

Adding self-adaptation capabilities to a software system introduces additional security risks, a problem that is not very well studied. In this paper, we proposed a runtime approach for vulnerability analysis of architecture-based adaptations. In this approach, attack models are generated for individual configurations, enabling the analysis of vulnerabilities and quantifying the security risks of configurations and adaptations. Then, the triggered adaptations are assessed from a security perspective to select a proper adaptation that is applied to the system according to the business policies. We implemented our approach in Rainbow and applied it on two cases to show its effectiveness.

As a future work, we plan to formalize the approach and apply it on more complex self-adaptive systems. We also plan to use the approach to construct on-the-fly cost-efficient countermeasures to mitigate attacks at runtime. Investigating more scalable threat modeling and analysis techniques for vulnerability analysis of self-adaptive systems is another research direction we plan to study.

Acknowledgment This work was supported by Swedish Knowledge Foundation (No. 20160186).

\section{REFERENCES}

[1] Hussain MJ Almohri, Layne T Watson, Danfeng Yao, and Xinming Ou. Security optimization of dynamic networks with probabilistic graph modeling and linear programming. IEEE Transactions on Dependable and Secure Computing, 13(4):474-487, 2016. 
[2] Mohamed Amoud and Ounsa Roudies. Dynamic adaptation and reconfiguration of security in mobile devices. In 2017 International Conference On Cyber Incident Response, Coordination, Containment Control (Cyber Incident), pages 1-6, June 2017.

[3] Shanai Ardi, David Byers, and Nahid Shahmehri. Towards a structured unified process for software security. In Proceedings of the 2006 International Workshop on Software Engineering for Secure Systems, SESS '06, pages 3-10, New York, NY, USA, 2006. ACM.

[4] Manuel Bertoa and Antonio Vallecillo. Usability metrics for software components. 042012.

[5] Radu Calinescu, Danny Weyns, Simos Gerasimou, Muhammad Usman Iftikhar, Ibrahim Habli, and Tim Kelly. Engineering trustworthy selfadaptive software with dynamic assurance cases. IEEE Trans. Software Eng., 44(11):1039-1069, 2018.

[6] Shang-Wen Cheng and David Garlan. Stitch: A language for architecture-based self-adaptation. Journal of Systems and Software, Special Issue on State of the Art in Self-Adaptive Systems, 85(12), December 2012. http://dx.doi.org/10.1016/j.jss.2012.02.060.

[7] Rogério de Lemos et al. Software engineering for self-adaptive systems: A second research roadmap. In Software Engineering for Self-Adaptive Systems, 2010

[8] S. Dobson, S. Denazis, and A. Fernández et al. A survey of autonomic communications. ACM Transactions on Autonomous and Adaptive Systems, 1(2):223-259, 2006.

[9] Ahmed M. Elkhodary, Naeem Esfahani, and Sam Malek. FUSION: a framework for engineering self-tuning self-adaptive software systems. In Proceedings of the 18th ACM SIGSOFT International Symposium on Foundations of Software Engineering, 2010, Santa Fe, NM, USA, November 7-11, 2010, pages 7-16, 2010.

[10] Betty H. C. Cheng et al. Software Engineering for Self-Adaptive Systems: A Research Roadmap, pages 1-26. Springer Berlin Heidelberg, Berlin, Heidelberg, 2009.

[11] Marcel Frigault, Lingyu Wang, Anoop Singhal, and Sushil Jajodia. Measuring network security using dynamic bayesian network. In Proceedings of the 4th ACM workshop on Quality of protection, pages 23-30. ACM, 2008.

[12] David Garlan, Shang-Wen Cheng, An-Cheng Huang, Bradley Schmerl, and Peter Steenkiste. Rainbow: architecture-based self-adaptation with reusable infrastructure. Computer, 37(10):46-54, Oct 2004.

[13] David Garlan, Robert T. Monroe, and David Wile. Acme: Architectural description of component-based systems. In Gary T. Leavens and Murali Sitaraman, editors, Foundations of Component-Based Systems, pages 47 68. Cambridge University Press, 2000.

[14] Jin Bum Hong and Dong Seong Kim. Towards scalable security analysis using multi-layered security models. Journal of Network and Computer Applications, 75:156 - 168, 2016.

[15] Nwokedi Idika and Bharat Bhargava. Extending attack graph-based security metrics and aggregating their application. IEEE Transactions on Dependable and Secure Computing, 9(1):75-85, Jan 2012.

[16] Jeffrey O. Kephart and David M. Chess. The vision of autonomic computing. Computer, 36(1):41-50, January 2003.

[17] Narges Khakpour, Farhad Arbab, and Éric Rutten. Supervisory controller synthesis for safe software adaptation. In 12th International Workshop on Discrete Event Systems, WODES 2014, Cachan, France, May 14-16, 2014., pages 39-45, 2014.

[18] Narges Khakpour, Farhad Arbab, and Éric Rutten. Synthesizing structural and behavioral control for reconfigurations in component-based systems. Formal Asp. Comput., 28(1):21-43, 2016.

[19] Narges Khakpour, Saeed Jalili, Carolyn L. Talcott, Marjan Sirjani, and Mohammad Reza Mousavi. Formal modeling of evolving self-adaptive systems. Sci. Comput. Program., 78(1):3-26, 2012.

[20] Barbara Kordy, Ludovic Piètre-Cambacédès, and Patrick Schweitzer Dag-based attack and defense modeling: Don't miss the forest for the attack trees. Computer Science Review, 13, 032013.

[21] Christiaan Johan Lamprecht. Adaptive Security. $\mathrm{PhD}$ thesis, Newcastle University, 2012.

[22] Wei Li and Rayford B. Vaughn. Cluster security research involving the modeling of network exploitations using exploitation graphs. In Sixth IEEE International Symposium on Cluster Computing and the Grid (CCGRID'06), volume 2, pages 26-26, May 2006.

[23] Lingyu Wang Marcel Frigault. Measuring network security using bayesian network-based attack graphs. In 2008 32nd Annual IEEE International Computer Software and Applications Conference, pages 698-703, July 2008
[24] Mark A. Flynn George A. Beitel Miles A. McQueen, Wayne F. Boyer. Quantitative cyber risk reduction estimation methodology for a small scada control system. In Proceedings of the 39th Annual Hawaii International Conference on System Sciences (HICSS'06), volume 9, pages 226-226, Jan 2006.

[25] Xinming Ou, Wayne F. Boyer, and Miles A. McQueen. A scalable approach to attack graph generation. In Proceedings of the 13th ACM Conference on Computer and Communications Security, CCS '06, pages 336-345, New York, NY, USA, 2006. ACM

[26] Xinming Ou, Sudhakar Govindavajhala, and Andrew W. Appel. Mulval: A logic-based network security analyzer. In Proceedings of the 14th Conference on USENIX Security Symposium - Volume 14, SSYM'05, pages 8-8, Berkeley, CA, USA, 2005. USENIX Association.

[27] Federico Quin, Danny Weyns, Thomas Bamelis, Sarpreet Singh Buttar, and Sam Michiels. Efficient analysis of large adaptation spaces in selfadaptive systems using machine learning. In International Symposium on Software Engineering for Adaptive and Self-Managing Systems. IEEE, 2019.

[28] Alex Ramos, Marcella Lazar, Raimir H. Filho, and Joel J. P. C. Rodrigues. Model-based quantitative network security metrics: A survey. IEEE Communications Surveys Tutorials, 19(4):2704-2734, Fourthquarter 2017

[29] M. Salehie and L. Tahvildari. Self-adaptive software: Landscape and research challenges. ACM Transactions on Autonomous and Adaptive Systems, 4(2):14:1-14:42, 2009.

[30] Goran Saman Nariman. A framework for secure structural adaptation. Master's thesis, Linnaeus University, Department of computer science and media technology, 2018

[31] Bradley Schmerl, Javier Cámara, Jeffrey Gennari, David Garlan, Paulo Casanova, Gabriel A Moreno, Thomas J Glazier, and Jeffrey M Barnes. Architecture-based self-protection: composing and reasoning about denial-of-service mitigations. In Proceedings of the 2014 Symposium and Bootcamp on the Science of Security, page 2. ACM, 2014.

[32] Chee-Wooi Ten, Govindarasu Manimaran, and Chen-Ching Liu. Cybersecurity for critical infrastructures: Attack and defense modeling. IEEE Transactions on Systems, Man, and Cybernetics-Part A: Systems and Humans, 40(4):853-865, 2010.

[33] Thein Than Tun, Mu Yang, Arosha Bandara, Yijun Yu, Armstrong Nhlabatsi, Niamul Khan, Khaled Khan, and Bashar Nuseibeh. Requirements and specifications for adaptive security: concepts and analysis. In Proceedings of the 13th International Conference on Software Engineering for Adaptive and Self-Managing Systems, SEAMS@ICSE 2018, Gothenburg, Sweden, May 28-29, 2018, pages 161-171, 2018.

[34] Hai L. Vu, Kenneth K. Khaw, Ty Chen, and Fei-Ching Kuo. A new approach for network vulnerability analysis. In 2008 33rd IEEE Conference on Local Computer Networks (LCN), pages 200-206, Oct 2008.

[35] Lingyu Wang, Tania Islam, Tao Long, Anoop Singhal, and Sushil Jajodia. An attack graph-based probabilistic security metric. In Vijay Atluri, editor, Data and Applications Security XXII, pages 283-296, Berlin, Heidelberg, 2008. Springer Berlin Heidelberg.

[36] D. Weyns. Software engineering for self-adaptive systems. In Handbook of Software Engineering. Springer, 2019.

[37] Eric Yuan, Naeem Esfahani, and Sam Malek. A systematic survey of self-protecting software systems. ACM Trans. Auton. Adapt. Syst., 8(4):17:1-17:41, January 2014.

[38] Eric Yuan, Sam Malek, Bradley Schmerl, David Garlan, and Jeff Gennari. Architecture-based self-protecting software systems. In Proceedings of the 9th International ACM Sigsoft Conference on Quality of Software Architectures, QoSA '13, pages 33-42, New York, NY, USA, 2013. ACM

[39] Simon Enoch Yusuf, Mengmeng Ge, Jin B. Hong, Hani Alzaid, and Dong Seong Kim. Evaluating the effectiveness of security metrics for dynamic networks. In 2017 IEEE Trustcom/BigDataSE/ICESS, Sydney, Australia, August 1-4, 2017, pages 277-284, 2017.

[40] Simon Enoch Yusuf, Mengmeng Ge, Jin Bum Hong, Huy Kang Kim, Paul Kim, and Dong Seong Kim. Security modelling and analysis of dynamic enterprise networks. In 2016 IEEE International Conference on Computer and Information Technology, CIT 2016, Nadi, Fiji, December 8-10, 2016, pages 249-256, 2016. 